\title{
SURFACE BRIGHTNESS FLUCTUATIONS AND THE STELLAR POPULATION IN ELLIPTICAL GALAXIES
}

\author{
MARIANNE SODEMANN AND BJARNE THOMSEN \\ Inst. of Phys. and Astron., Aarhus Univ., DK-8000 Aarhus C. \\ E-mail: ms@obs.aau.dk, bt@obs.aau.dk
}

The Surface Brightness Fluctuation (SBF) method introduced by Tonry \& Schneider (1988) is known as a distance indicator. However, we focus on another aspect. Our aim is to obtain new information on the stellar population that constitutes an elliptical galaxy by comparing radial gradients in SBF magnitudes with traditional broad-band colours. We have obtained I-band images of NGC 3379 with the NTT/SUSI, 0!"8 - 1".2 FWHM, Sodemann \& Thomsen (1994). An analysis similar to that of Tonry \& Schneider (1988) is applied. However, the smoothed galaxy is obtained from (Thomsen \& Baum, 1989)، For NGC 3379 we find a SBF signal of $3.39 \pm 0.02 \mathrm{ADU}$, see Figure 1, corresponding to an object with apparent magnitude $\bar{I}=28 \mathrm{~m} \cdot 62 \pm 0 \mathrm{~m} \cdot 03$. If the image is subdivided into 4 annular regions, we find the gradient in $\bar{I}$ in Figure 2. As both fluctuation 'noise' and photon noise raise towards the centre of the galaxy, it is difficult to correct for globular clusters in a position independent way. Dealing with gradients of the SBF signal, the task is even more delicate. The weakening of $\bar{I}$ towards the galaxy centre is foreseen by the stellar population models of Worthey (1994).

\section{References}

Sodemann, M., Thomsen, B. (1994) Astron. Astrophys. (in press)

Thomsen, B., Baum, W.A. (1989) Astrophys. J., 347, 214

Tonry, J., Schneider, D.P. (1988) Astron. J., 96, 807

Worthey, G. (1994) Astrophys. J. Suppl. (in press)
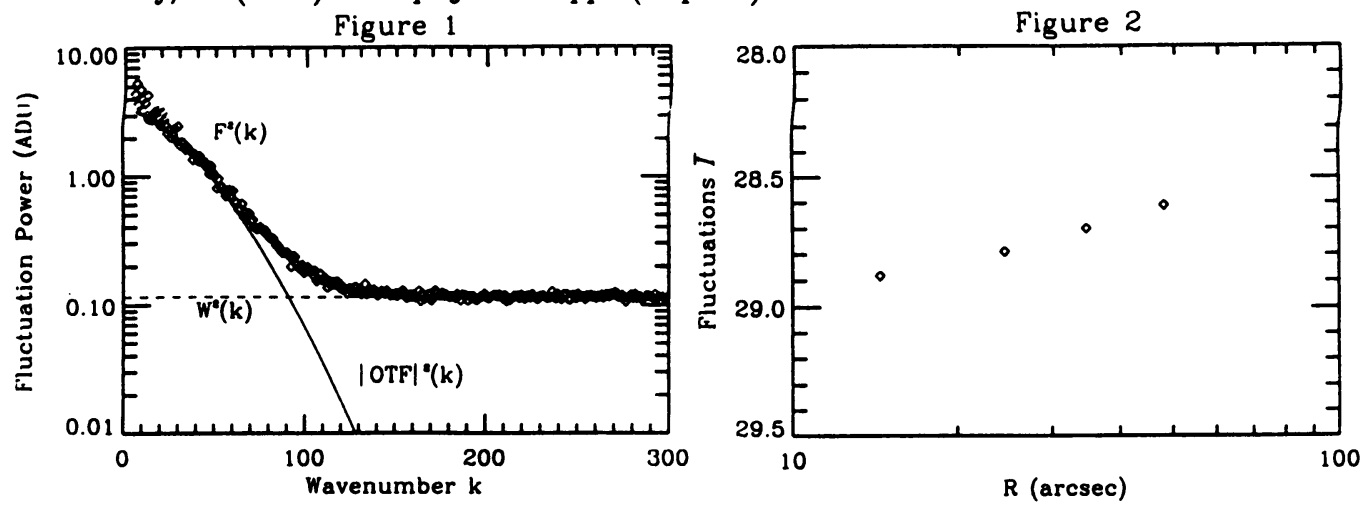